Open Access

\title{
The impact of colors on human memory in learning English collocations: evidence from south Asian tertiary ESL students
}

\author{
Jahangeer Khan ${ }^{*}$ (D) and Chengyu Liu
}

\author{
* Correspondence: \\ jahangirkhan850@ymail.com \\ We would like to express our \\ sincere gratitude to Prof. Li Heng at \\ the College of International Studies, \\ Southwest University, China for his \\ academic assistance in the research \\ and then to the South Asian \\ colleagues and other international \\ students at Southwest University, \\ China for their contributions, \\ particularly in the process of data \\ collection and analysis for the \\ current paper despite their tough \\ study schedules. \\ College of International Studies, \\ Southwest University, Tiansheng \\ Road, Beibei, Chongqing, China
}

\begin{abstract}
This paper aims to highlight the significance of color that captivates human attention in learning English collocations in an educational setting. In order to expedite the impact of color on memory, an empirical research was conducted, in which 30 South Asian ESL students of a local university in China were selected as participants, equally divided into the experimental and the control groups. All the participants were taught literal and context-based meanings of 30 English collocations for three consecutive days. A pre-test and a post-test were administered to assess the impact of color on the students' working memory in learning English collocations. Prior to assessing the impact of color on memory, the participants in the experimental group were exposed to the underlined collocations as written on colorful papers (yellow), while same collocations were imprinted on simple white color papers for the controlled group. A t-test was administered for the statistical analysis of the data. Comparatively, in the post-test, the participants in the experimental group remarkably outperformed while retrieving English collocations and their meanings by the virtue of yellow color. The finding of the current paper lends support to the notion that color, as an environmental stimulus, may greatly affect human working memory in learning English collocations. The study is expected to provide implications to text and curriculum designers by supplementing ESL learners with colorful texts and images in order to harness their language efficiencies and performances.
\end{abstract}

Keywords: English collocations, Color, Working memory, South Asian tertiary ESL students

\section{Introduction}

Working memory is an enthralling area of psycholinguistics, yet it goes through certain complex learning processes in retrieving different images, colors and vocabulary. Even, the process becomes more complex if color is taken into consideration as an external stimulus for memory in the learning processes of adults and children. The current paper deals with the trilateral association of color, memory and English collocations particularly to examine how effectively human memory retrieves collocations with the

(c) The Author(s). 2020 Open Access This article is licensed under a Creative Commons Attribution 4.0 International License, which permits use, sharing, adaptation, distribution and reproduction in any medium or format, as long as you give appropriate credit to the original author(s) and the source, provide a link to the Creative Commons licence, and indicate if changes were made. The images or other third party material in this article are included in the article's Creative Commons licence, unless indicated otherwise in a credit line to the material. If material is not included in the article's Creative Commons licence and your intended use is not permitted by statutory regulation or exceeds the permitted use, you will need to obtain permission directly from the copyright holder. To view a copy of this licence, visit http://creativecommons.org/licenses/by/4.0/. 
help of color. At this juncture, we assume that if the occurrence of collocation takes place inside the memory, and color, on the other hand, triggers the arousal and retrieval processes of memory, then color may affect collocation within the same framework of memory. Nevertheless, our current study does not merely rely on the general perception of color in correspondence to working memory; rather, we expedite how warm type of color such as yellow stimulates learners' memory in the recall process of acquiring English collocations. In this frame of reference, previous studies (e.g. Hanna \& Remington, 1996) suggest that color can optimize learners' efficiencies in retrieving a big chunk of information and knowledge productivity (i.e. collocations, phrases and idioms) instantaneously. By the same token, color can enhance learners' attention and levitate their levels of memory retention and recognition (Dzulkifli \& Mustafar, 2013). Despite the fact that human cognitive system involves multifaceted processes such as memory, perception and attention, yet it became an enthralling area for many psycholinguists to investigate the association of color, attention and memory performance for the effective pedagogical implications. A plethora of studies revealed the paramount importance of colors that trigger human working memory in the learning process. In this regard, Chang, $\mathrm{Xu}$, and Watt (2018) argued that colors play a significant role in reducing cognitive overload in learning process. Therefore, psycholinguists recommend the inclusion of words with colorful background in textbooks for the curtailment of such cognitive overload to optimize learning goals. Furthermore, color-coding techniques and color cues help various psychologists to identify and treat aged patients who suffer from language disabilities such as dementia and autism (Cernin, Keller, \& Stoner, 2003). Under the pedagogical domain, familiarity with color naming process inside texts further on provides enormous opportunities to ESL learners by learning various idiomatic expressions and collocations such as bright eyes and pale face standing for hope and fear, red cheeks and brown hair standing for beauty and glamour. In this frame of reference, the current research is not only theoretically necessary to bridge the empirical gap pertinent to the effect of color on memory of English vocabulary but is also practically significant for enhancing the mastery of English vocabulary by changing the way in which English words and expressions are presented visually in teaching materials as well as in classroom presentation. Therefore, our current paper is designed to investigate how the selected South Asian ESL learners get command over English collocations they are exposed to with questionnaires having yellow color overlays in the post-test.

\section{Literature review}

A variety of studies have been conducted by adopting multiple approaches to accentuate how deeply colors may affect human memory to recall various words (as well as their collocations) and images that pass through from Short-term Memory (STM) to Long-term Memory (LTM).

In order to rationalize and justify the impact of color on memory, it is desirable to extend our study to the mechanism of working memory that operates on certain principles in the retrieval process of information. Verily, it is reasonable to say that the function of memory is a complex phenomenon in retrieving information which needs a profound critical analysis of cognitive science. In this context, it is significant to comprehend how deeply the process of information takes place in memory rather than how 
recently the information takes place (Zahedi \& Abdi, 2012). Thereby, Craik and Lockhart (1972) presented the "depth of processing" theory that working memory contains STM and LTM; the former retains acoustically repeated words and collocation for a short time, while the latter exhibits images, faces and semantic mapping for a long time inside the memory.

The same view was further on supported by Baddeley (2000) by elaborating that the function of STM is to hold memory traces for some time before they fade along with a mechanism for rehearsal of articulation which reactivates the traces. Another difference between STM and LTM is the frequency of words which explicitly affects long-term memory rather than short-term retention (Glanzer, 1972, p.166). In terms of retention of information inside the memory chamber, Craik and Lockhart (1972) proposed that STM retains information for $30 \mathrm{~s}$ while LTM does the same process for a minute to a year, whereas a large number of scholars also favor the notion that the retrieval of information inside the human memory largely depends upon the presented time to which learners are exposed to.

Apart from the functionality of human memory, Hsu (2007) argued that the term collocation has been derived from the Latin word "collocare" which refers to arrange or sit in order. In simple terms, collocation is a combination of words which transpire systematically. The conceptual framework of collocation, for the first time in the twentieth century, was given by Firth who argued that collocation is a company of words that continuously occurs in a habitual way (Léon, 2007, p.404). Gradually, a large community of linguists then supported Firth's claim when new discoveries transpired into this field. Some other scholars such as Ellis, Simpson-Vlach, and Maynard (2008) argued that the production of collocation emerges from learners' acquired association of concepts that occur in human memory. Such types of concepts generally develop prefabricated expressions in the form of collocation which could be retrieved from the memory at once (Saudin, 2015). Our current study may not cover up a wide range of scholastic views in terms of what types of collocations take place inside the memory, rather, it aims to undertake the central role of color as an external factor by stimulating working memory to retrieve various collocations.

In this regard, our current assertion which consolidates the effect of colors on working memory is based on the theoretical framework presented by Dzulkifli and Mustafar (2013) who argued that color and memory are closely interlinked and that color plays a central role in the retrieval process of words and images. The claim got a considerable support from the empirical observations carried out by Smilek, Dixon, Cudahy, and Merikle (2002) to assess the effect of color on memory. Participants were exposed to numerical digits with four colors namely black, white, congruent and incongruent for three minutes. After a stipulated time, participants in the experimental group outperformed by identifying colorful digits in a recall test which suggests that color directly affects human memory. So far, scholars have defined colors from their own points of view in context of working memory, however, the concept of color as presented by Hanna and Remington (1996) seems very concise and compatible by demonstrating that color is a sign which conveniently cultivates learners' efficiency in retrieving information.

However, in the case of toddlers' acquisition of lexical items, the role of color seems less effective as their vocabulary recognition capacities largely depend on their 
preliminary knowledge, experience and shapes of objects to which they are exposed to by their parents (Perry \& Saffran, 2017). Under the domain of pedagogical facet, advanced learners of ESL look at various colorful images for general observations to learn vocabulary, yet they need to take into account these colors for idiomatic and metaphorical expressions as well such as bright eyes and yellow face which stand for hope and fear, while pink cheeks and red lips stand for glamour (Donaghy \& Xerri, 2017, p.74). Another construct of the same domain is the significance of color that varies from culture to culture. Gibson et al. (2017) suggests that color naming is highly associated with its usefulness in a particular culture. For instance, while red color in the Chinese culture is perceived as a sign of valor, courage and revolution, white color stand for peace and stability in the Pakistani community. Therefore, such familiarity with color levitates not only the linguistic competence of ESL learners but also helps them to be acquainted with the significance of colors in diverse cultures. However, the significance of colors for language instructions and learning further on raises certain questions to identify which type of color is more or less effective in the retention and recognition process of information so far.

To date, a plethora of studies indicates that warm colors considerably affect attention and arousal which cause to increase memory's performance than cool colors. According to Dzulkifli and Mustafar (2013) emotional arousal is a psychological or physiological alertness which is the outcome of various colors in our surrounding environment. Greene, Bell, and Boyer (1983) also argues that warm colors such as red, yellow and orange have a greater effect on human memory in retaining information than cool colors such as brown and grey. This concept was also supported by Rogahang, Liando, and Maru (2016) by stating that warm colors are vivid and look prominent in a natural environment than cool colors which are soothing and recede. The empirical study of Krahn (2018) supports the same claim that red color retains high accuracy in the recognition memory whereas a majority of the participants recalled red color more quickly than black and blue colors during the experiment. In this regard, if color attracts human attention, and attention causes to create arousal, then, certainly color optimizes memory performance (ibid). In order to recall previous information more quickly, learners are needed to get maximum exposure to colorful texts as color is an effective environmental stimulus to increase memory's performance (Farley \& Grant, 1976).

Thus, the robust influence of colorful images on memory can boost up learners' academic performance and level of motivation. Wichmann et al. (2002) also argues that colorful images channelize information to the cognitive system much faster than colorless images and thus improve memory performance. This idea was also supported by Hanna and Remington (1996) who argued that well-learned colorful objects can increase accuracy and recognition speed of memory in an explicit way. Correspondingly, some studies also suggest that words which are associated with each other in our mental lexicon occur due to the sight and visionary stimulation which help learners to link them with similar shapes and objects that have already existed in their minds due to their previous exposures to similar shapes (Nattinger, 1988, p.64).

So far, under the auspices of various scholastics views, one thing became translucent that the production of collocations, shapes and images precisely occurs in human memory whether it occurs on account of priming, high and low frequencies or adjacencies of words whereas color, as an environmental stimulus, plays a key role in retrieving 
similar information. In the latter part of the paper, we shall empirically expedite that how warm color capacitates human memory in learning English collocations in a recall test.

\section{Methodology}

The purpose of our current paper is to investigate the impact of color on memory in learning collocation. In order to achieve this goal, we administer closed-ended questionnaires as a research instrument in pre- and post-tests categorically.

\section{Participants}

Forty $(N=40)$ South Asian male students, ranging from 25 to 35 years old, were selected randomly from different fields of master studies at Southwest University, China. The majority of them were from Afghanistan, Pakistan and India, where English was their medium of instruction in their previous schooling (see Table 1 for some additional information of participants). An English proficiency pre-test was administered in the form of closed-ended questionnaire (30 English collocations), which brought 30 successful participants for the next stage (post-test). These participants were then divided into two groups, i.e. the controlled group and the experimental group.

\section{Research instruments}

In order to evaluate an optimal result in the data collection process, the researcher designed a closed-ended questionnaire containing 30 collocations with four options (see the Appendix). The questionnaire was aimed to collect objective data from the respondents. However, colored overlays of questionnaires for both groups were different in pre-test and post-test, i.e. collocations were written on simple white papers for both groups in the pre-test, while same collocations were imprinted on colorful papers (yellow) for the experimental group in the post-test. The design of questionnaire was simple with clear-cut instructions on the top of the page for both groups (also see the Appendix). All the given questions were relevant in accordance with our given hypotheses.

\section{Procedure}

At the initial stage, a pre-test containing a close-ended questionnaire (MCQ format) was administered in order to select a homogenized number of participants in order to assess the participants' English language proficiency skill. There were 30 MCQs (collocations) on the white sheet with four options where participants were instructed to

Table 1 Basic information of the participants

\begin{tabular}{ll}
\hline No of Samples & 30 \\
\hline Gender & Male only \\
Age & $25-35$ years \\
Major & Journalism, mathematics, psychology and English literature \\
Level of Education & Master and PhD \\
Citizenship & Afghanistan, Pakistan and India \\
Mother tongue & Pashto, Urdu and Dari \\
\hline
\end{tabular}


mark only one correct answer. Each correct question carried 1 mark while negative marking criteria were avoided. The allocated time for the test was $20 \mathrm{~min}$. The reliability and passing criteria of the test were based on allocated marks (20/30). By administering a $t$-test, we came across that only 30 out of the 40 participants who secured 20 and above marks were qualified for the post-test.

Table 2 indicates that both groups possessed the similar level of knowledge of English collocations they were exposed to with no significant difference.

At this stage, the probability value is 0.71 , which is greater than 0.05 and hereby indicates that our null hypothesis is correct in the pre-test (i.e. the proficiency levels of respondents in both groups are almost similar).

Based on their secured marks, they were divided into two groups (i.e. controlled and experimental) with a ratio of 15:15 in each group.

It is noticeable that before administering the post-test, the colors of questionnaires for both groups were different, i.e. yellow color for the experimental group while simple white color for the controlled group. The same English collocations were written for both groups. The participants in both groups were taught the literal and contextual meanings of these collocations with numerous examples for three consecutive days into three different sessions whereas each session lasted for 10-15 min. After an interval of two weeks, a post-test was administered to assess the impact of color on working memory in learning English collocations to which participants in the experimental group were exposed to. The highest obtained scores in the experimental group were 30, while in the controlled group the highest obtained scores were 29. The format of the posttest and the pre-test was similar (i.e. $30 \mathrm{MCQs}$ in the form of English collocations, see the Appendix).

\section{Results and analysis}

By administering the post-test, we observed that participants in the experimental group outperformed to the controlled group after attempting MCQs in questionnaires which had yellow background color by retrieving the exact meaning of English collocations even after an interval of two weeks. The following Table 3 indicates a comparative statistical analysis of both groups by employing $t$-test.

Table 3 indicates that the mean averages of both the experimental and controlled groups tend to demonstrate a minute difference (i.e. the mean average of the experimental group is 25.6 while that of the controlled group is 23.73). However, there is a minute difference between the standard deviations of both groups by the ratio of 2.26 and 2.57 . Noteworthy to say that the probability value that we achieved is 0.04 , which is less than 0.05 and hereby indicates that our null hypothesis is wrong (i.e. participants in the experimental group outperformed to the controlled group in retrieving English collocations conveniently in the post-test).

Table 2 Distribution of Pre-test scores of both groups to select participants for the post-test

\begin{tabular}{lllll}
\hline Groups & $\mathrm{N}$ & Mean & Std. Deviation & Probability Value \\
\hline Experimental group & 15 & 24.06 & 2.43 & $P<0.71$ \\
Controlled group & 15 & 23.73 & 2.57 & \\
\hline
\end{tabular}


Table 3 Distribution of Post-test scores of both experimental and controlled groups

\begin{tabular}{lllll}
\hline Groups & $\mathrm{N}$ & Mean & Std. Deviation & Probability Value \\
\hline Experimental group & 15 & 25.60 & 2.26 & $P<0.04418493$ \\
Controlled group & 15 & 23.73 & 2.57 & \\
\hline
\end{tabular}

\section{Discussion}

Our empirical data provides some sort of predictability of our given assumption in terms of retrieving English collocations via colorful questionnaires in the experimental group. A large number of respondents in the experimental group opted for maximum correct collocations on yellow type questionnaires even after an interval of two weeks (Obtained marks in the experimental group were ascending from 21 till 30 resulting probability value 0.044 ).

Similarly, our claim sought out a consolidated support from the empirical data presented by Cui, Gao, Zhou, and Guo (2016) who argued that colors retain a long-term effect on recognition memory through familiarity and recollection processes of certain episodic events. Even, some empirical studies indicate that surface information and early visual processing of colored images are more effective than black-and-white colored images to retention and recognition processes (Homa \& Viera, 1988). However, our main concern is not to measure the impact of monochromatic and polychromatic colors rather to pinpoint the overwhelming impact of warm colors on retention and recognition capacities of human memory during learning process. In short, our obtained statistical result in the post-test coincides with the theoretical framework suggested by Dzulkifli and Mustafar (2013) by illustrating the effect of color on working memory in retaining English collocations through exposures.

As far as language instructions are concerned, our current paper also derives our readers towards a wide range of pedagogical implications that could be carried out in the future by obtaining effective learning goals, including: (1) The impact of color on memory maximizes ESL learners' efficiencies without spending much time in searching of form and meaning of lexical bundles and collocations. (2) Owing to cultural diversity, some ESL learners e.g. Chinese and South Asian learners may encounter cognitive overburden in memorizing a text. Consequently, this study lends support to various curriculum and text designers to add up texts with colorful background in textbooks for decreasing cognitive overburden of learners, particularly at the tertiary level of education. (3) Based on a brief study of working memory, this paper suggests that ESL instructors need to optimize learning opportunities by adapting and modifying multiple language strategies in the contemporary curricula so as to meet the required pedagogical goals, e.g. memory, cognitive and meta-cognitive strategies.

\section{Conclusion}

Based on a thorough theoretical and empirical framework of the study, it became intelligible that color affects human memory which causes to captivate various words and images in the recall process. The statistical analysis of the collected data accentuates that human mind tends to captivate comfortably words and images that are transcribed on colorful pages under a controlled environment. Undoubtedly, word retention is a complicated process which requires a comprehensive study to cover up the entire mental framework categorically such as LTM and STM. Thereupon, our empirical data 
indicates that human mind attracts prominent and visible entities and colors much easier than other ordinary environmental inputs in the learning process.

Apart from the fact that warm types of color influence memory recognition and recalling processes, Singg and Mull (2017) further on proposed that learning effect of color relies on genders' category i.e. red and yellow may have greater impact on male while cool color such as blue could exert greater influence on female. Therefore, on account of time constraints, our current study is limited in terms of involuntary avoidance of female participants during the experiment.

Additionally, our current study provides future implications to our prospective readers and research aspirants to explore and identify some additional factors in a similar research paradigm i.e. physiological and socio-cognitive factors, equal participation of gender, a combination of contrastive colors of text with overlay colors of pages, classroom setting, the font size and font face of the text, the attitude of language instructors, the learners' background knowledge and exposure to English as L2.

In a similar pattern, ESL learners should be taught effectively how to use colors in formulating idiomatic expressions and collocations via related colorful images such as white cheeks, pale face, and brown hair. Considering the cultural diversity of learning environments, language instructors, text and curriculum designers are expected to represent colorful texts and learning materials which should be compatible and acceptable in accordance to the learners' perceptual knowledge of colors in their own cultures.

\section{Appendix}

Please tick only one appropriate answer for each of the following collocations. Each question carries one mark. The total allocated time is $20 \mathrm{~min}$.

\begin{tabular}{|c|c|c|c|}
\hline 1. Heavy Workload & & 2) Heavy Feature & \\
\hline a) Overload & b) Less Burden & a) Dumb & b) Deaf \\
\hline c) Lazy & d) Smart & c) Prominent Quality & d) Simple \\
\hline 3) Heavy Rain & & 4) Heavy Sleeper & \\
\hline a) Too much Rain & b) Thundering & a) Active one & b) Who sleeps too late \\
\hline c) Snow Fall & d) Sunny day & c) Awakening too early & d) Lazy one \\
\hline 5) Back Pay & & 6) Balance Diet & \\
\hline a) Demand of money & b) Refusal of money & a) Unhealthy diet & b) Oily diet \\
\hline c) Giving up efforts & d) Wandering & c) Healthy diet & d) None of them \\
\hline 7) Bad Habit & & 8) Great Strength & \\
\hline a) Good Habit & b) Option "a" & a) Weakness & b) Valor \\
\hline c) Nasty Habit & d) None of them & c) Frail & d) All of them \\
\hline 9) Native Speaker & & 10) Strong Coffee & \\
\hline a) A Stranger & b) Naïve speaker & a) Pure coffee & b) Impure coffee \\
\hline c) Original inhabitant & d) Multilingual & d) Salty coffee & d) None of them \\
\hline 11) Express Service & & 12) Rich History & \\
\hline a) Slow service & b) Nasty service & a) Alive history & b) Dead history \\
\hline c) Fast Service & d) All of them & c) Both "a"\&"b" & d) None of them \\
\hline 13) Abuse of Power & & 14) Pay Attention & \\
\hline a) Unethical use of power & b) Ethical use of power & a) Focus & b) Look around \\
\hline c) Having less power & d) Neutral position & c) Ignorance & d) None of them \\
\hline
\end{tabular}


Appendix (Continued)

\section{5) Make an Effort}

a) Delay a task

c) Concentration

\section{7) Go Bankrupt}

a) Become wealthy

c) Idle

\section{9) Core Value}

a) Less valuable

c) Basic value

21) Quite Agree

a) Complete consent

c) Ignore quietly

23) Furious about
a) Enraged
c) Think about

25) Break the silence
a) Keep quite
b) Get angry
C) Say something suddenly
d) Option B

\section{7) Get a Chance}

a) Avail an opportunity

c) Loss of work

b) Miss an opportunity

d) None of them

\section{6) Get Angry}
a) Enraged
b) Happy
c) Make Noise
d) Both B, C

\section{8) Keep Quite}
a) Be silent
b) Be crazy
c) Be smart
d) Option B

\section{0) Irreparable Damage}
a) Full coverage
b) Irreversible loss
c) Partial loss
d) None of them

\section{2) Apologize for}
a) Get angry
b) Say sorry
c) Walk slowly
d) None of them

24) Break the rules
a) Disobey the rules
b) Accept challenges
c) Run away
d) None of them

26) Come close
a) Near
b) Far away
c) At a long distance
d) Run away

\section{8) Give a call}
a) Give a ring
b) Reminder
c) Stop
d) Ignorance

\section{0) Have a Chat}
a) Mad
b) Polite
a) Start Talking
b) Stop talking
c) silent
d) Sneezing
d) Wondering

Abbreviations

STM: Short-term Memory; LTM: Long-term Memory

Acknowledgments

We would like to express our sincere gratitude to Prof. Li Heng at the College of International Studies, Southwest University, China for his academic assistance in the research and then to the South Asian colleagues and other international students at Southwest University, China for their contributions, particularly in the process of data collection and analysis for the current paper despite their tough study schedules.

Authors' contributions

Both the authors, namely JK and LC, offered their sincere efforts in data collection and analysis processes. More specifically, the research contribution made by the co-author LC is really inspiring in terms of providing theoretical insight and making grammatical correction in the current manuscript. The authors read and approved the final manuscript.

Funding

The funding for the collection, analysis and interpretation of the data was provided by the authors themselves.

Availability of data and materials

The authors of the current manuscript are willing to reproduce materials and data if required.

\section{Competing interests}

The authors declare that they have no competing interests.

Received: 28 March 2020 Accepted: 17 August 2020

Published online: 10 September 2020

\section{References}

Baddeley, A. D. (2000). The episodic buffer. A new component of working memory? Trends in Cognitive Sciences, 4(11), 417- 
Cernin, P. A., Keller, B. K., \& Stoner, J. A. (2003). Color vision in Alzheimer's patients: Can we improve object recognition with color cues? Aging, Neuropsychology, and Cognition, 10(4), 255-267.

Chang, B., Xu, R., \& Watt, T. (2018). The impact of colors on learning A paper presented at Adult Education Research Conference 2018, University of Victoria, Canada, June 7-10. Available at https://www.researchgate.net/publication/33584 0864_The_Impact_of_Colors_on_Learning.

Craik, F. I., \& Lockhart, R. S. (1972). Levels of processing: A framework for memory research. Journal of Verbal Learning and Verbal Behavior, 11(6), 671-684.

Cui, X., Gao, C., Zhou, J., \& Guo, C. (2016). Can color changes alter the neural correlates of recognition memory? Manipulation of processing affects an electrophysiological indicator of conceptual implicit memory. NeuroReport, 27(14), 1037-1045.

Donaghy, K., \& Xerri, D. (2017). The image in English language teaching (pp. 1-11). Floriana: ELT Council.

Dzulkifli, M. A., \& Mustafar, M. F. (2013). The influence of colour on memory performance: A review. The Malaysian Journal of Medical Sciences, 20(2), 3-9.

Ellis, N. C., Simpson-Vlach, R. I. T. A., \& Maynard, C. (2008). Formulaic language in native and second language speakers: Psycholinguistics, corpus linguistics, and TESOL. TESOL Quarterly, 42(3), 375-396.

Farley, F. H., \& Grant, A. P. (1976). Arousal and cognition: Memory for color versus black and white multimedia presentation. The Journal of Psychology, 94(1), 147-150.

Gibson, E., Futrell, R., Jara-Ettinger, J., Mahowald, K., Bergen, L., Ratnasingam, S., \& Conway, B. R. (2017). Color naming across languages reflects color use. Proceedings of the National Academy of Sciences, 114(40), 10785-10790.

Glanzer, M. (1972). Storage mechanisms in recall. In G. H. Bower (Ed.), The psychology of learning and motivation: Advances in research and theory. New York: Academic Press. 5, 129-193.

Greene, T. C., Bell, P. A., \& Boyer, W. N. (1983). Coloring the environment: Hue, arousal, and boredom. Bulletin of the Psychonomic Society, 21(4), 253-254.

Hanna, A., \& Remington, R. (1996). The representation of color and form in long-term memory. Memory \& Cognition, 24(3), $322-330$.

Homa, D., \& Viera, C. (1988). Long-term memory for pictures under conditions of thematically related foils. Memory \& Cognition, 16(5), 411-421.

Hsu, J. Y. (2007). Lexical collocations and their impact on the online writing of Taiwanese college English majors and non-English majors Online Submission.

Krahn, E. (2018). Decomposing the effect of color on memory: How red and blue affect memory differently.

Léon, J. (2007). Meaning by collocation. In History of Linguistics 2005: Selected Papers from the Tenth International Conference on History of the Language Sciences (ICHOLS X), 1-5 September 2005, Urbana-Champaign, Illinois (Vol. 112), (p. 404). Philadelphia / Amsterdam: John Benjamins publishing.

Nattinger, J. (1988). Some current trends in vocabulary teaching. Vocabulary and Language Teaching, 1, $62-82$.

Perry, L. K., \& Saffran, J. R. (2017). Is a pink cow still a cow? Individual differences in toddlers' vocabulary knowledge and lexical representations. Cognitive Science, 41(4), 1090-1105.

Rogahang, L. A., Liando, N. V., \& Maru, M. G. (2016). The impact of colors on SMA Negeri 1 Tomohon student's immediate and delayed retention of vocabulary. Journal of English Language and Literature Learning, 1(1), 41-55.

Saudin, H. (2015). Collocation: Theoretical considerations, methods and techniques for teaching it.

Singg, S., \& Mull, C. W. (2017). Effect of color on information retention by young men and women. JOJ Case Stud, 2(4), 555591.

Smilek, D., Dixon, M. J., Cudahy, C., \& Merikle, P. M. (2002). Synesthetic color experiences influence memory. Psychological Science, 13(6), 548-552.

Wichmann, F. A., Sharpe, L. T., \& Gegenfurtner, K. R. (2002). The contributions of color to recognition memory for natural scenes. Journal of Experimental Psychology: Learning, Memory, and Cognition, 28(3), 509.

Zahedi, Y., \& Abdi, M. (2012). The impact of imagery strategy on EFL learners' vocabulary learning. Procedia - Social and Behavioral Sciences, 69, 2264-2272.

\section{Publisher's Note}

Springer Nature remains neutral with regard to jurisdictional claims in published maps and institutional affiliations.

\section{Submit your manuscript to a SpringerOpen ${ }^{\circ}$ journal and benefit from:}

- Convenient online submission

- Rigorous peer review

- Open access: articles freely available online

- High visibility within the field

- Retaining the copyright to your article

Submit your next manuscript at $\mathbf{s p r i n g e r o p e n . c o m ~}$ 